\title{
Performance Enhancement of SOVA Based Decoder in SCCC and PCCC Schemes
}

\author{
Ahmed A. Hamad \\ Department of Electrical Engineering, University of Babylon, Babel, Iraq. \\ Email: ahmedbabel@yahoo.com \\ Received October $13^{\text {th }}, 2012$; revised November $20^{\text {th }}, 2012$; accepted December $9^{\text {th }}, 2012$
}

\begin{abstract}
This study proposes a simple scaling factor approach to improve the performance of parallel-concatenated convolutional code (PCCC) and serial concatenated convolutional code (SCCC) systems based on suboptimal soft-input soft-output (SISO) decoders. Fixed and adaptive scaling factors were estimated to mitigate both the optimistic nature of a posteriori information and the correlation between intrinsic and extrinsic information produced by soft-output Viterbi (SOVA) decoders. The scaling factors could be computed off-line to reduce processing time and implementation complexity. The simulation results show a significant improvement in terms of bit-error rate (BER) over additive white Gaussian noise and Rayleigh fading channel. The convergence properties of the suggested iterative scheme are assessed using the extrinsic information transfer (EXIT) chart analysis technique.
\end{abstract}

Keywords: Turbo Codes; SOVA; SCCC; Scaling Factor; EXIT

\section{Introduction}

Since the invention of turbo codes [1], a considerable interest had been devoted to reduce the complexity of the optimum MAP [2] decoding algorithm. This is to comply with the excessive need for low-power and low-cost decoder chips that are used in wireless mobile devices that pervade in recent years. The log-MAP, max-log-MAP, and SOVA algorithms [3-5] are some of the examples proposed for this target. The reduction in complexity that is achieved by near optimum algorithms like SOVA is accompanied by degradation in performance in terms of bit error rate.

Several papers have looked into the reasons behind the degradation in performance of these practical turbo-decoding algorithms, especially the one based on SOVA relative to the MAP algorithm [6-12]. A conviction has been created that the reason behind this degradation is the overestimation of reliability values generated by the SOVA decoder compared by those that would have been produced by the MAP decoder. It has been suggested in some of those papers that this optimistic extrinsic information may be due to the relatively high correlation between the intrinsic and extrinsic information [6]. As a result, various approaches were suggested to improve the performance of SOVA. Fixed and adaptive scaling factors are the more conventional approaches that are used to alleviate the distortion of the extrinsic information produced by SOVA [11].
This study proposes a simple approach for dealing with the exaggerated reliability values and the excessive correlation between the intrinsic and extrinsic information produced by the SOVA decoder. The suggested remedy is based on mathematical statistics, and it involves using two scaling factors, one is applied to the a-posteriori soft-output information of the SOVA and another is applied to the extrinsic information before it passes to the other decoder component in the iterative decoding process.

It is worth mentioning that the proposed approach could be applied to both SCCC and PCCC schemes based on MAP, log-MAP, max-log-MAP and SOVA decoders and it has almost the same complexity as that of the conventional schemes, which makes it quite attractive. The numerical results show that the turbo decoding algorithm based on SOVA that employs the proposed scaling factors can achieve a better performance compared to the one that does not employ scaling factors.

\section{Improved SOVA}

\subsection{PCCC Scheme}

In this work, it has been suggested that the turbo code consists of two recursive systematic convolutional codes (RSC) joined by an interleaver.

Let $u=\left[u_{1}, u_{2}, \cdots, u_{k}\right]$ where $u \in\{0,1\}$, is the binary information sequence. The modulated symbols corre- 
sponding to the coded bits are as follows

$$
c=\left[c_{1,1}, c_{1,2}, c_{1,3}, \cdots, c_{k, 1}, c_{k, 2}, c_{k, 3}\right],
$$

where $c \in\{-1,+1\}$, assuming binary phase shift keying modulation is applied. The noisy received sequence at the channel output is

$$
y=\left[y_{1,1}, y_{1,2}, y_{1,3}, \cdots, y_{k, 1}, y_{k, 2}, y_{k, 3}\right]
$$

and it is given by;

$$
y_{k, i}=d_{k, i} c_{k, i}+\omega_{k, i} \quad i=1,2,3
$$

where $d_{k, i}$ is the fading magnitude, and $\omega_{k, i}$ is additive noise modeled as Gaussian with zero mean and variance $N_{o} / 2$ (the two-sided power spectral density of the Gaussian channel). When the simulation is used over the additive white Gaussian channel (AWGN), $d_{k, i}$ is 1 , while it is a Rayleigh random variable for the case of a Rayleigh flat-fading channel. It is assumed that coherent detection with a perfect estimation of $d_{k, i}$ is present at the receiver.

To avoid extra complexity when using the MAP algorithm, the SOVA decoder is proposed. Actually, SOVA has two essential modifications (over the conventional Viterbi Algorithm) which allows it to be used as a component decoder for turbo codes [4,13]. Firstly, the path metrics used are modified to take account of a-priori information when selecting the maximum likelihood path through the trellis. Secondly, the algorithm is modified so that it provides a soft output in the form of the a-posteriori LLR $\left(L_{a p}\right)$ for each decoded bit. After each iteration, the LLR for first decoder $\left(L_{a p}\right)$ can be represented as [14]

$$
L_{a p}=L_{c} y_{k, 1}+L_{a}+L_{e}
$$

where $L_{c} y_{k, 1}$ is the received systematic symbol scaled by the reliability of the channel $L_{c} \quad\left(L_{c}=4 d r \frac{E_{b}}{N_{o}}\right.$, which can be set to 1 for SOVA [15]), where $r$ is the rate of the code, $E_{b}$ is the energy per information bit, and $L_{a}$ is the a priori information achieved by interleaving ( $\Pi$ ) or deinterleaving $\left(\Pi^{-1}\right)$ of the extrinsic information $L_{e}$ produced by the other decoder. For the first decoder (DEC1), $L_{a}^{1}=\Pi^{-1}\left[L_{e}^{2}\right]$, whereas for second decoder (DEC2), $L_{a}^{2}=\Pi\left[L_{e}^{1}\right]$.

To improve the reliability of $L_{a p}$ for practical decoders like SOVA, two scaling factor $\alpha_{1}$ and $\alpha_{2}$ are proposed. The scaled $L_{a p}$ is given by

$$
\hat{L}_{a p}^{j}=\alpha_{j} L_{a p}^{j} \quad j=1,2 .
$$

The values of $\alpha_{1}$ and $\alpha_{2}$ are derived based on the minimum mean-square error (MMSE) criterion [16] as follows.

The threshold value of $\hat{L}_{a p}^{1}$, i.e., $\operatorname{sgn}\left(\hat{L}_{a p}^{1}\right)$ produced by $\mathrm{DEC} 1$ is a growing estimate to the systematic coded bits $c_{k, 1}$ with each iteration. Statistically, their meansquare difference (MSD)

$$
\delta\left(\alpha_{1}\right)=E\left\{\left[\alpha_{1} \operatorname{sgn}\left(L_{a p}^{1}\right)-c_{k, 1}\right]^{2}\right\},
$$

is a measure of how efficient the algorithm is with the proposed modification. $E[\cdot]$ denotes the expected value.

In a similar sense, the MSD related to DEC2 is

$$
\delta\left(\alpha_{2}\right)=E\left\{\left[\alpha_{2} \operatorname{sgn}\left(L_{a p}^{2}\right)-\Pi\left[c_{k, 1}\right]\right]^{2}\right\} .
$$

It is obvious that to get better suboptimal decoding, the parameter $\alpha_{j}$ should be found to minimize the MSD $\delta\left(\alpha_{j}\right)$, which means that $\mathrm{d} \delta\left(\alpha_{j}\right) / \mathrm{d} \alpha_{j}=0$. From this equation, $\alpha_{1}$ and $\alpha_{2}$ are found to be

$$
\alpha_{1}=E\left[\operatorname{sgn}\left(L_{a p}^{1}\right) \cdot c_{k, 1}\right] \text {, }
$$

and

$$
\alpha_{2}=E\left[\operatorname{sgn}\left(L_{a p}^{2}\right) \cdot \Pi\left[c_{k, 1}\right]\right],
$$

given that

$$
E\left\{\left[\operatorname{sgn}\left(L_{a p}^{j}\right)\right]^{2}\right\}=1 \text {. }
$$

We can describe the correlation between the received sequence and extrinsic information by their correlation coefficient [16]. Therefore, we have two correlation coefficients as follows

$$
\rho_{1}\left(y_{k, 1}, L_{e}^{1}\right)=\frac{\boldsymbol{E}\left\{\left(y_{k, 1}-\boldsymbol{E}\left[y_{k, 1}\right]\right)\left(L_{e}^{1}-\boldsymbol{E}\left[L_{e}^{1}\right]\right)\right\}}{\sqrt{\boldsymbol{E}\left[\left(y_{k, 1}-\boldsymbol{E}\left[y_{k, 1}\right]\right)^{2}\right] \boldsymbol{E}\left[\left(L_{e}^{1}-\boldsymbol{E}\left[L_{e}^{1}\right]\right)^{2}\right]}},
$$

$$
\begin{aligned}
& \rho_{2}\left(\Pi\left(y_{k, 1}\right), L_{e}^{2}\right) \\
= & \frac{E\left\{\left(\Pi\left(y_{k, 1}\right)-E\left[\Pi\left(y_{k, 1}\right)\right]\right)\left(L_{e}^{2}-E\left[L_{e}^{2}\right]\right)\right\}}{\sqrt{E\left[\left(\Pi\left(y_{k, 1}\right)-E\left[\Pi\left(y_{k, 1}\right)\right]\right)^{2}\right] E\left[\left(L_{e}^{2}-E\left[L_{e}^{2}\right]\right)^{2}\right]}} .
\end{aligned}
$$

To reduce the correlation between intrinsic and extrinsic information, it is proposed that we scale $L_{e}^{j}$ by $\beta_{j}$

$$
\hat{L}_{e}^{j}=\beta_{j} L_{e}^{j},
$$

where

$$
\beta_{j}=\left(1-\rho_{j}\right) .
$$

The above method can be implemented as shown in Figure 1.

\subsection{SCCC Scheme}

A similar approach is considered for the case of the SCCC scheme with little modification. A simple block diagram for the conventional SCCC encoder and modi- 
fied decoder is depicted in Figure 2. Simulation results show that better performance can be achieved by scaling the LLRs which are produced by outer decoder (ODEC) as shown in Figure 2(b).

Following a similar procedure to PCCC, the LLR immediate produces by the ODEC scaled as:

$$
\alpha_{2}=E\left\{\operatorname{sgn}\left(L_{a p}^{2}\right) \cdot c_{k}^{o}\right\} \text {. }
$$

Whereas, the extrinsic information for the same decoder is scaled by $\beta_{2}$ given by Equation (10). Here, the correlation coefficient $\rho_{2}$ may be represented as

$$
\rho_{2}\left(L_{a}^{2}, L_{e}^{2}\right)=\frac{E\left\{\left(L_{a}^{2}-E\left[L_{a}^{2}\right]\right)\left(L_{e}^{2}-E\left[L_{e}^{2}\right]\right)\right\}}{\sqrt{E\left[\left(L_{a}^{2}-E\left[L_{a}^{2}\right]\right)^{2}\right] E\left[\left(L_{e}^{2}-E\left[L_{e}^{2}\right]\right)^{2}\right]}} .
$$

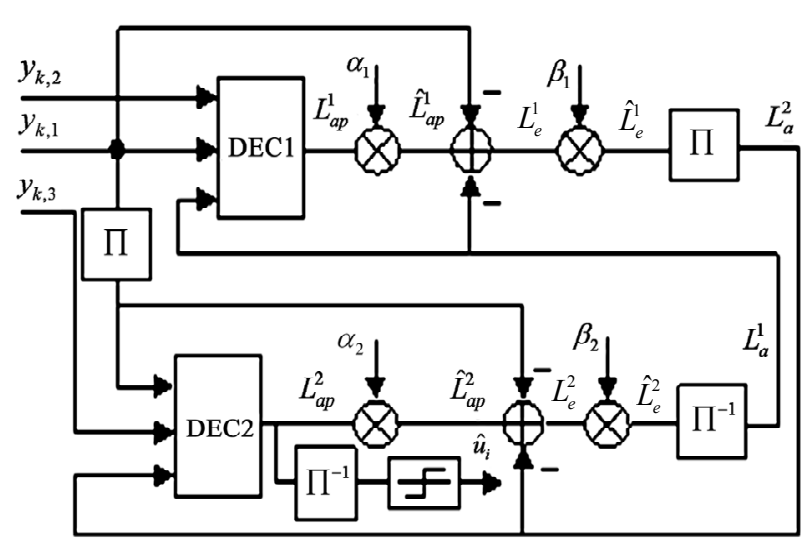

Figure 1. Implementation of modified SOVA.

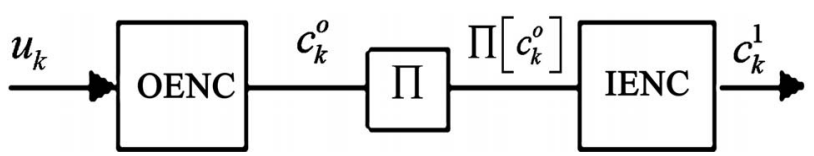

(a)

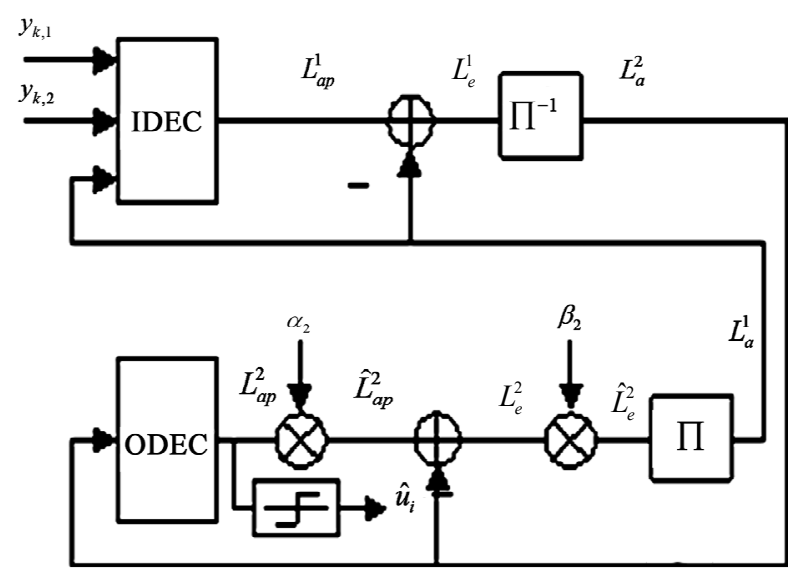

(b)

Figure 2. (a) SCCC encoder, (b) Modified SCCC decoder.

\section{Numerical Results}

This section presents the effectiveness of scaling factors on the performance of PCCC and SCCC schemes. The simulations were implemented over AWGN and flat fading Rayleigh channels. For the fading channel, the sampling time of the input signal is taken to be $(1 / 50000)$ sec. and maximum Doppler shift of $100 \mathrm{~Hz}$.

The $1 / 3$ rate turbo code that is specified for high-speed downlink packet access (HSDPA) in UMTS [17] is considered here which consists of two $1 / 2$ rate component RSC codes of memory 3 , with polynomials $(\mathrm{Gr}, \mathrm{Gf})=(1+$ $\mathrm{D} 2+\mathrm{D} 3,1+\mathrm{D}+\mathrm{D} 3)$. The two RSCs are joined by interleaver of sizes 1024 bits, which is constructed for the same standard [17]. In SCCC, the same constituent codes are used, and to obtain a total coding rate of $1 / 3$, the coded bits have been puncture using the pattern $[10,11]$. To examine the effectiveness of the four scaling factors ( $\alpha_{j}$ and $\beta_{j}, j=1,2$ ), different combinations of these factors are considered. Figures 3 and $\mathbf{4}$ show the BER

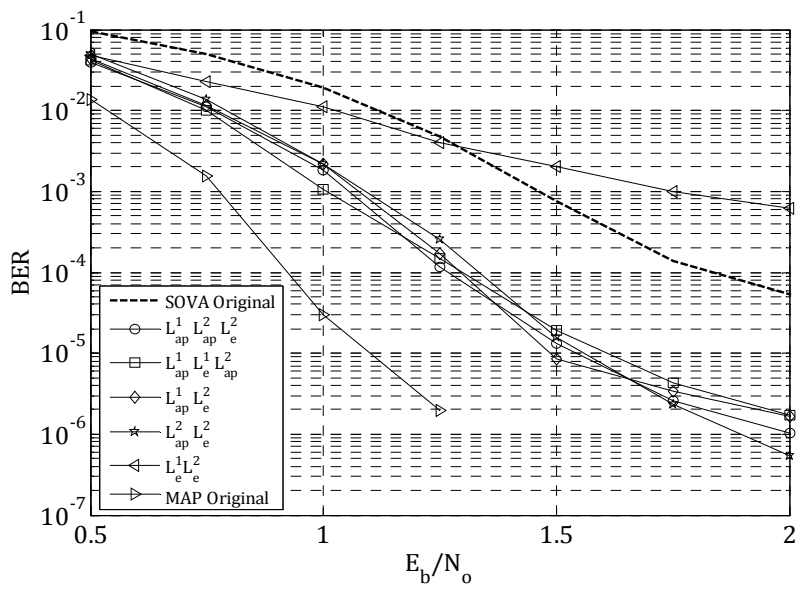

Figure 3. BER performance of modified PCCC systems over AWGN channel.

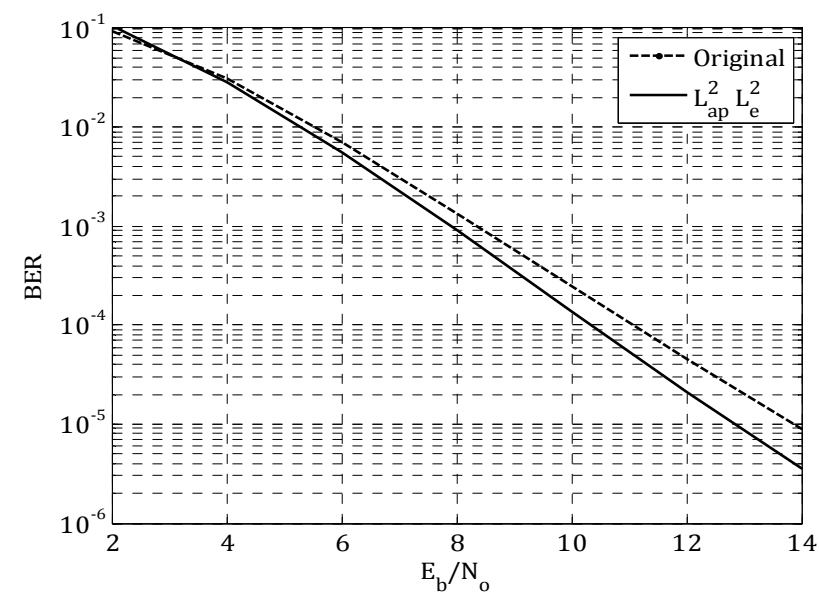

Figure 4. BER performance of modified system $L_{a p}^{2} L_{e}^{2} \quad$ over Rayleigh fading channel. 
results for the proposed PCCC systems after five iterations over AWGN and Rayleigh fading channels, respectively. To avoid congestion, each figure is restricted to a number of curves, which demonstrate better improvement. The proposed schemes are also compared with the performance of turbo decoder based on log-MAP algorithm simulated over AWGN channel. Each curve is labeled with a group of symbols, which describe the simulated system. The letters $L_{a p}$ and $L_{e}$ refer to the scaled LLR and the numeral superscript indicates which decoder is belong (DEC1 or DEC2). The letters " $\mathrm{O}$ " and "I" refer to the outer (ODEC) and inner (IDEC) decoders respectively.

The simulation presents the gain achieved (about 0.9 $\mathrm{dB}$ over AWGN and $1 \mathrm{~dB}$ over faded channel at BER of $10^{-5}$ ) by modified systems (with scaling) in comparison with the original system (without scaling). The perform- ance of modified systems are become closer by about $0.35 \mathrm{~dB}(\sim 0.75 \mathrm{~dB}$ for unmodified systems $)$ to the performance of turbo code utilizes log-MAP decoder at BER of $10^{-4}$. Table 1 presents the average scaling factors $\left(\alpha_{2}\right.$ and $\beta_{2}$ ) derived offline for five iterations to system $L_{a p}^{2} L_{e}^{2}$ at different signal to noise ratios $\left(E_{b} / N_{o}\right)$ over flat fading channels. Referring to Equation (6) and (7), the values of $\alpha_{j}$ should be estimated offline, because the systematic coded bits $\left(c_{k, 1}\right)$ are not available at the decoder side, whereas the values of $\beta_{j}$ can be obtained online. Utilizing the simulation results taken from the "free-running" iterative decoder, the average trajectory of the extrinsic information transfer chart (EXIT) [18] for system $\left(L_{a p}^{1} L_{a p}^{2} L_{e}^{2}\right)$ is depicted in Figure 5 at $E_{b} / N_{o}=$ $0.5 \mathrm{~dB}$. In comparison to the trajectory of original system, it is obvious from Figure 5 that the modified systems present better convergence in mutual information.

Table 1. Average scaling factors, $\alpha_{2}$ and $\beta_{2}$ applied on system $L_{a p}^{2} L_{e}^{2}$ for different $E_{b} / N_{o}$ and 5 iterations assuming Rayleigh fading channel.

\begin{tabular}{|c|c|c|c|c|c|c|c|c|c|c|}
\hline \multicolumn{11}{|c|}{$L_{a p}^{2} L_{e}^{2}$} \\
\hline$E_{b} / N_{o}(\mathrm{~dB})$ & & & $\alpha_{2}$ & & & & & $\beta_{2}$ & & \\
\hline & Iter1 & Iter2 & Iter3 & Iter4 & Iter5 & Iter1 & Iter2 & Iter3 & Iter4 & Iter5 \\
\hline 2.0 & 0.769 & 0.754 & 0.785 & 0.766 & 0.784 & 0.948 & 0.791 & 0.847 & 0.784 & 0.830 \\
\hline 4.0 & 0.893 & 0.925 & 0.937 & 0.939 & 0.943 & 0.636 & 0.542 & 0.538 & 0.518 & 0.521 \\
\hline 6.0 & 0.959 & 0.982 & 0.987 & 0.988 & 0.989 & 0.490 & 0.437 & 0.424 & 0.416 & 0.412 \\
\hline 8.0 & 0.986 & 0.996 & 0.998 & 0.998 & 0.998 & 0.426 & 0.390 & 0.379 & 0.374 & 0.371 \\
\hline 10.0 & 0.996 & 0.999 & 1.000 & 1.000 & 1.000 & 0.394 & 0.365 & 0.356 & 0.353 & 0.351 \\
\hline 12.0 & 0.999 & 1.000 & 1.000 & 1.000 & 1.000 & 0.375 & 0.351 & 0.343 & 0.340 & 0.339 \\
\hline 14.0 & 1.000 & 1.000 & 1.000 & 1.000 & 1.000 & 0.364 & 0.342 & 0.335 & 0.332 & 0.332 \\
\hline
\end{tabular}

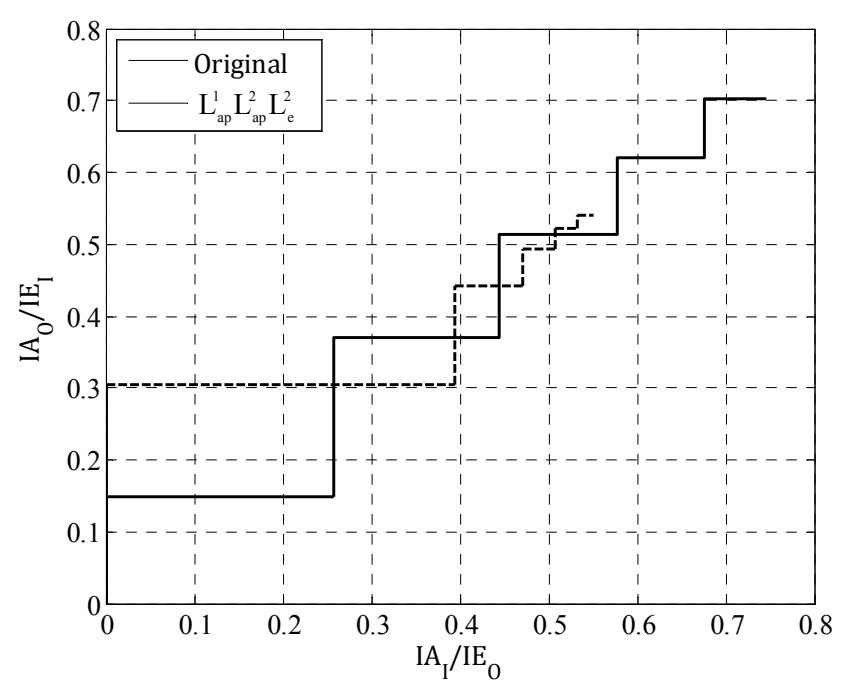

Figure 5. Average trajectory for system $\left(L_{a p}^{1} L_{a p}^{2} L_{e}^{2}\right)$ at $E_{b} / N_{o}=0.5 \mathrm{~dB}$ in AWGN channel. 


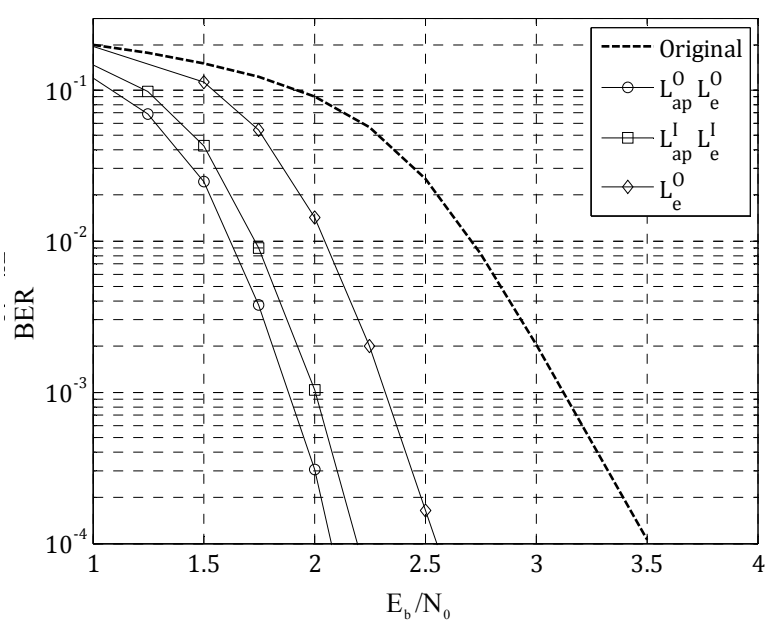

Figure 6. BER performance of modified SCCC systems over AWGN channel.

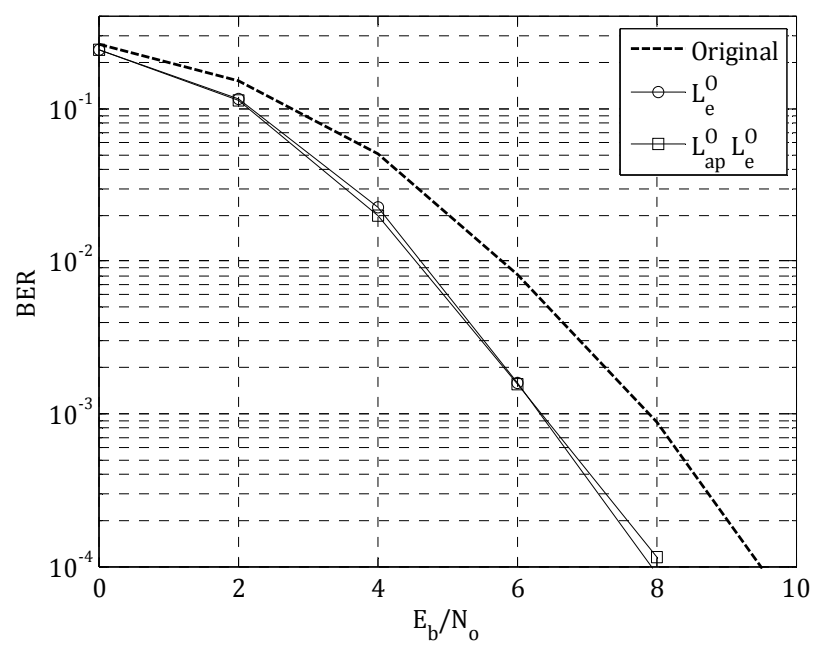

Figure 7. BER performance of modified SCCC systems over Rayleigh fading channel.

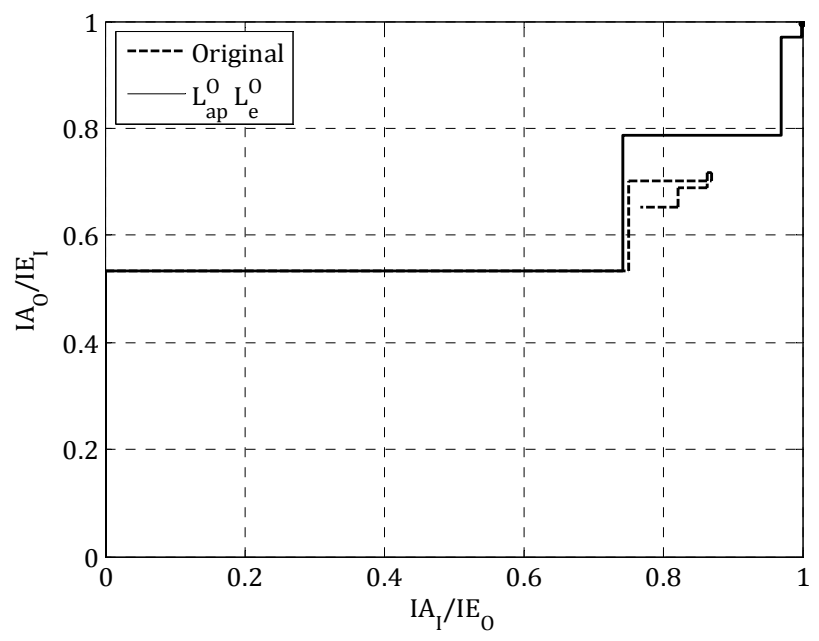

Figure 8. Average trajectory for system $\left(L_{a p}^{o} L_{e}^{o}\right)$ at $E_{b} / N_{o}$ $=2.25 \mathrm{~dB}$ in AWGN channel.
Figures 6 and 7 show the BER performance of modified SCCC schemes compared with the original SOVA over AWGN and flat fading channel respectively. The simulation of scheme $L_{a p}^{o} L_{e}^{o}$ reveals similar improvement of about $1.5 \mathrm{~dB}$ at BER $10^{-4}$ over the two channels. Figure 8 shows a stall in the mutual information trajectory for conventional SOVA after two iterations, whereas $L_{a p}^{o} L_{e}^{o}$ continue in converge.

\section{Conclusion}

This paper introduces simple modifications to the conventional SOVA to alleviate the effect of optimistic a posteriori information and the strong correlation between the input and output of the SOVA. A method for offline and online computation of scaling factors has also been described. It has shown that the proposed scheme is significantly improved the performance of PCCC and SCCC schemes that are based on suboptimal decoders. The simulation shows that better improvement could be achieved by adding two or three simple multipliers to the traditional PCCC scheme, whereas two multipliers are sufficient to produce a modified SCCC scheme. The complexity resulting from incorporating scaling factors is almost the same as that of the traditional method without these factors. The convergence behavior of such decoders is investigated by using extrinsic information transfer (EXIT) charts.

\section{REFERENCES}

[1] C. Berrou, A. Glavieux and P. Thitimajshima, "Near Shannon Limit Error-Correcting Coding and Decoding: Turbo Codes," Proceedings of the International Conference on Communications, Geneva, 23-26 May 1993, pp. 10641070.

[2] J. Hagenauer and P. Robertson, "Iterative ("Turbo") Decoding of Systematic Convolutional Codes with the MAP And SOVA Algorithm," Proceedings of the ITG Tagung, Codierung für Quelle, Kanal and Ubertragung, Frankfurt, October 1994, pp. 21-29.

[3] P. Robertson, E. Villebrun and P. Höher, "A Comparison of Optimal and Sub-Optimal MAP Decoding Algorithms Operating in the Log Domain," Proceedings of the International Conference on Communications, Seattle, 18-22 June 1995, pp. 1009-1013.

[4] J. Hagenauer and P. Hoeher, "A Viterbi Algorithm with Soft-Decision Outputs and Its Applications," Proceedings of IEEE Global Telecommunications Conference, Dallas, 27-30 November 1989, pp. 1680-1686.

[5] C. Berrou, P. Adde, E. Angui and S. Faudeil, "A Low Complexity Soft-Output Viterbi Decoder Architecture," Proceedings of IEEE International Conference on Communications, Geneva, Vol. 2, 23-26 May 1993, pp. 737740.

[6] L. Papke, P. Robertson and E. Villerbrun, "Improved De- 
coding with the SOVA in a Parallel Concatenated (Turbo-Code) Scheme," Proceedings of the IEEE International Conference Communications (ICC), Dallas, 23-27 June 1996, pp. 102-106.

[7] C. H. Wang, W. T. Wang and C. C. Chao, "A Unified Structure of Trellis-Based Soft-Output Decoding Algorithms for Turbo Codes," IEEE Transactions on Communications, Vol. 52, No. 8, 2004, pp. 1355-1366. doi:10.1109/TCOMM.2004.833025

[8] G. Colavolpe, G. Ferrari and R. Raheli, "Extrinsic Information in Iterative Decoding: A Unified View," IEEE Transactions on Communications, Vol. 49, No. 12, 2001, pp. 2088-2094. doi:10.1109/26.974255

[9] C. X. Huang and A. Ghrayeb, "A Simple Remedy for the Exaggerated Extrinsic Information Produced by SOVA Algorithm," IEEE Transactions on Wireless Communications, Vol. 5, No. 5, 2006, pp. 996-1002. doi:10.1109/TWC.2006.1633352

[10] S. Papaharalabos, P. Sweeney, B. G. Evans and P. T. Mathiopoulos, "Improved Performance SOVA Turbo Decoder," IEE Proceedings-Communications, Vol. 153, No. 5, 2006, pp. 586-590. doi:10.1049/ip-com:20050247

[11] C. X. Huang and A. Ghrayeb, "Improved SOVA and APP Decoding Algorithms for Serial Concatenated Codes," IEEE Globecom, Dallas, 29 November-3 December 2004, pp. 189-193.
[12] D.-W. Yue and H. H. Nguyen, "Unified Scaling Factor Approach for Turbo Decoding Algorithms," IET Communications, Vol. 4, No. 8, 2010, pp. 905-914. doi:10.1049/iet-com.2009.0125

[13] C. Berrou, P. Adde, E. Angui and S. Faudeil, "A Low Complexity Soft-Output Viterbi Decoder Architecture," Proceedings of ICC 1993, Geneva, May 1993, pp. 737740.

[14] J. Hagenauer, E. Offer and L. Papke, "Iterative Decoding of Binary Block and Convolutional Codes," IEEE Transactions on Information Theory, Vol. 42, No. 2, 1996, pp. 429-445. doi:10.1109/18.485714

[15] L. Lin and R. S. Cheng, "Improvements in SOVA-Based Decoding for Turbo Codes," Proceedings of the IEEE International Conference on Communications (ICC), Montreal, Vol. 3, 8-12 June 1997, pp. 1473-1478.

[16] A. Papoulis, "Probability, Random Variables, and Stochastic Processes," McGraw-Hill Inc., Montreal, 1991.

[17] 3GPP, “Ts 25.212 v3.11.0 (2002-09)," Technical Specification Group Radio Access Network, Multiplexing and Channel Coding (FDD), 1999.

[18] S. T. Brink, "Convergence Behavior of Iteratively Decoded Parallel Concatenated Codes," IEEE Transactions on Communications, Vol. 49, No. 10, 2001, pp. 1727-1737. doi:10.1109/26.957394 